\title{
Negative regulation of Armadillo, a Wingless effector in Drosophila
}

\author{
Li-Mei Pai ${ }^{1}$, Sandra Orsulic ${ }^{1, *}$, Amy Bejsovec ${ }^{2}$ and Mark Peifer ${ }^{1, \dagger}$ \\ ${ }^{1}$ Department of Biology and Curriculum in Genetics and Molecular Biology, University of North Carolina, Chapel Hill, NC 27599- \\ 3280, USA \\ 2Department of Biochemistry, Molecular Biology and Cell Biology, Northwestern University, Evanston IL 60208, USA \\ *Present address: Max-Planck Institüt für Immunbiologie, D-79108 Freiberg, Germany \\ †Corresponding author (e-mail: peifer@unc.edu)
}

\section{SUMMARY}

Drosophila Armadillo and its vertebrate homolog $\beta$-catenin play essential roles both in the transduction of Wingless/Wnt cell-cell signals and in the function of cellcell adherens junctions. Wingless and Wnts direct numerous cell fate choices during development. We

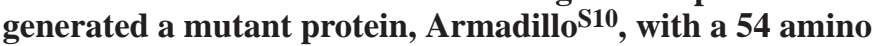
acid deletion in its N-terminal domain. This mutant is constitutively active in Wingless signaling; its activity is independent of both Wingless signal and endogenous wild-type Armadillo. Armadillo's role in signal transduction is normally negatively regulated by Zeste-white 3 kinase, which modulates Armadillo protein stability. Armadillos10 is more stable than wild-type Armadillo, suggesting that it is less rapidly targeted for degradation. We show that Armadillo $^{\text {S10 }}$ has escaped from negative regulation by Zeste white-3 kinase, and thus accumulates outside junctions even in the absence of Wingless signal. Finally, we present data implicating kinases in addition to Zeste white-3 in Armadillo phosphorylation. We discuss two models for the negative regulation of Armadillo in normal development and discuss how escape from this regulation contributes to tumorigenesis.

Key words: Armadillo, $\beta$-catenin, Wingless, Wnt, Zeste white-3, Drosophila

\section{INTRODUCTION}

During embryonic development, cells acquire information from many sources about the fates they should choose. One key source of information is communication among neighboring cells, which induces new cell fates and maintains old ones. The Wingless/Wnt family of cell-cell signaling proteins directs numerous processes, including anterior-posterior patterning of the Drosophila embryonic epidermis, dorsal-ventral patterning in early vertebrate embryos, and dorsal-ventral patterning of the limbs of flies and vertebrates (reviewed by Parr and McMahon, 1994).

Components of the Drosophila Wingless (Wg) signal transduction pathway have been identified; similar proteins also transduce certain vertebrate Wnt signals (reviewed by Orsulic and Peifer, 1996a). One component, Armadillo (Arm), accumulates both in adherens junctions, where it regulates cell adhesion (Cox et al., 1996), and in the cytoplasm and nucleus of certain cells, where it mediates $\mathrm{Wg}$ signal transduction. In our current model, soluble Armadillo (or its vertebrate homolog $\beta$-catenin) is rapidly degraded in the absence of $\mathrm{Wg} / \mathrm{Wnt}$ signal, and thus its steady state level outside adherens junctions is low (Peifer et al., 1994a; van Leeuwen et al., 1994). This degradation requires the action of both the serine/threonine (Ser/Thr) kinase Zeste white-3 (Zw3; its vertebrate homolog is glycogen synthase kinase 3- $\beta$ (GSK); Peifer et al., 1994a; Siegfried et al., 1994) and the product of the tumor suppressor protein adenomatous polyposis coli (APC; Munemitsu et al., 1995; Rubinfeld et al., 1996). In contrast, Wg interaction with its receptor (Bhanot et al., 1996) activates Dishevelled (Yanagawa et al., 1995), which counteracts Zw3 activity (Cook et al., 1996). This stabilizes Arm in the cytoplasm and nucleus (Orsulic and Peifer, 1996b).

The next step remained mysterious until recently. The first clue came from the realization that vertebrate $\beta$-catenin can form a complex with DNA-binding proteins of the TCF/LEF family, and these proteins could, when ectopically expressed in Xenopus, alter dorsal-ventral patterning in a way which suggested that they play a role in Wnt signaling (Behrens et al., 1996; Molenaar et al., 1996; Huber et al., 1996). We and others recently extended this work in Drosophila, showing that the fly TCF family member $d T C F$ plays an essential role in Wingless signaling in vivo (van de Wetering et al., 1997; Brunner et al., 1997). The active transcription factor is a complex between Arm and $d T C F$, and this complex directly regulates the expression of Wingless-responsive genes (van de Wetering et al., 1997; Riese et al., 1997).

In the experiments described here, we focused on one aspect of the signaling pathway, namely the means by which Zw3 negatively regulates Arm via effects on Arm stability. Since Zw3 is a Ser/Thr kinase, Arm degradation may be regulated by phosphorylation. Two possible Zw3 targets have been identified. One is Arm itself. Hypophosphorylated Arm accumulates in zw3 mutants (Peifer et al., 1994b). A Zw3/GSK phosphory- 
lation consensus site is conserved between Arm and $\beta$-catenin (Peifer et al., 1994b); GSK phosphorylates $\beta$-catenin in vitro, using this conserved site (Yost et al., 1996). Another possible target of Zw3/GSK is APC; GSK phosphorylates APC, promoting binding of APC to $\beta$-catenin and ultimately $\beta$ catenin degradation (Rubinfeld et al., 1996; Munemitsu et al., 1995). Regulation of Arm/ $\beta$-catenin degradation not only plays a key role in $\mathrm{Wg} / \mathrm{Wnt}$ signaling, but also is a target for activation in both colon cancer and melanoma (Korinek et al., 1997; Morin et al., 1997; Rubinfeld et al., 1997).

Here we report that deletion of the GSK/Zw3 consensus phosphorylation site in the $\mathrm{N}$-terminal domain of Arm relieves the normal negative regulation by Zw3, constitutively activating Arm in Wg signal transduction. This mutant Arm no longer depends on Wg signal nor on endogenous wild-type Arm for activity, and thus it not only escapes negative regulation but also can itself signal. Mutant protein accumulates at high levels in the cytoplasm and nucleus of all cells, regardless of whether they receive $\mathrm{Wg}$, mirroring the accumulation of wild-type Arm in a $z w 3$ mutant. Consistent with this, mutations in $z w 3$ do not increase the stability of the mutant Arm. The pool of Arm protein in adherens junctions remains fully phosphorylated in a zw3 mutant, suggesting that alternate kinases can phosphorylate Arm and raising the possibility that Zw3 may affect Arm phosphorylation indirectly by altering its stability. We discuss two alternative roles for Zw3 in Arm regulation.

\section{MATERIALS AND METHODS}

\section{armadillo mutants and other fly stocks}

Mutations were introduced into a c-myc-tagged arm cDNA (Orsulic and Peifer, 1996b; details upon request). arm $^{S 10}$ (a.a. 34-87 deleted) was created using an in vitro mutagenesis kit (USB) as recommended by the manufacturer. Wild-type or mutant cDNAs were introduced into the pUAST vector (Brand and Perrimon, 1993). $\operatorname{arm}^{\Delta N}$ (a.a. 1128 deleted) is described by Pai et al. (1996). $y \mathrm{arm}^{+} w$ flies were used for transformation. Several homozygous lines of each construct were established; mutant protein expression was tested by immunoblotting. $\mathrm{arm}^{Y D 35}, \mathrm{arm}^{H 8.6}$ and $\mathrm{arm}^{X P 33}$ are described by Peifer and Wieschaus (1990), $z w 3^{M 11-1}$ is described by Siegfried et al. (1994), and $w g^{I G 22}$ is described by Nüsslein-Volhard and Wieschaus (1980). Germline clones were produced as described by Peifer et al. (1994a). Most GAL4 stocks were from the Bloomington Drosophila stock center; en-GAL4 was provided by A. Brand.

\section{Genetic tests of function}

We performed four genetic tests of $\mathrm{arm}^{S 10}$ at $25^{\circ} \mathrm{C}$, using a line that expresses mutant protein at approximately wild-type levels. Progeny were examined as follows. Eggs were collected and hatch rates determined, cuticles of hatched larvae and unhatched embryos were prepared, and adult progeny were scored for viability. First, we tested $\mathrm{arm}^{S 10}$ in a zygotic null arm background, by crossing $\mathrm{arm}^{Y D 35} / F M 7$; $e 22 c-G A L 4 /+$ females to $\mathrm{arm}^{S 10}$ homozygous males. Second and third, we tested $\mathrm{arm}^{S 10}$ in embryos with a maternal and zygotic contribution composed entirely of $\operatorname{arm}^{X P 33}$ or $\operatorname{arm}^{H 8.6}$ mutant protein. Germline clones of $\mathrm{arm}^{X P 33}$ or arm $^{H 8.6}$ were generated as described by Peifer et al. (1994a). Females carrying germline clones and heterozygous for $e 22 c-G A L 4$ were mated to arm $^{S 10}$ homozygous males. Fourth, we tested $\mathrm{arm}^{S 10}$ in a $\mathrm{wg}$ background, by analyzing progeny of the recombinant stock $\mathrm{arm}^{S 10} \mathrm{wg}^{I G 22} / \mathrm{CyO}$. Cuticles were prepared as described by Wieschaus and Nüsslein-Volhard (1986).

\section{Biochemical and cell biological studies}

We used monoclonal anti-c-myc (purified from 9E10 cell supernatant; Orsulic and Peifer, 1996b; 1:200 for immunofluorescence and unpurified culture supernatant for immunoblotting), polyclonal anti-Arm N2 (Peifer et al., 1994a; 1:200), polyclonal anti-Arm CT (1:40), monoclonal anti-Arm 7A1 (Peifer et al., 1994a; 1:500), monoclonal antiEngrailed (DiNardo et al., 1985; 1:50) and polyclonal anti-Wg (van den Heuvel et al., 1989; 1:500). Immunoprecipitation, immunoblotting and cell fractionation were as in Peifer (1993). When immunoblotting, proteins were detected either by ECL (Amersham) or by using alkaline phosphatase-coupled secondary Ab, NBT and BCIP (Promega), as recommended by the manufacturers. Con ASepharose fractionation using NET buffer was as described by Pai et al. (1996). Okadaic acid and PP-2A treatment were as described by Peifer et al. (1994b). Protein localization in situ: (1) anti-myc antibody; as in Orsulic and Peifer (1996b), (2) anti-Arm; as in Peifer et al. (1991), (3) anti-Engrailed and Anti-Wg; as in DiNardo et al. (1985).

\section{RESULTS}

\section{Sequences in Armadillo's N-terminal domain negatively regulate Armadillo activity in Wingless signaling}

We previously characterized a number of arm mutant transgenes under the control of the arm promoter, and thus expressed ubiquitously at high levels (Orsulic and Peifer, 1996b). During those experiments we generated a mutation, $\mathrm{arm}^{\mathrm{S} 10}$, in which 54 amino acids of the $\mathrm{N}$-terminal domain were deleted (a.a. 34-87; Fig. 1A). Despite repeated efforts, we were unable to obtain transformants expressing $\mathrm{arm}^{\mathrm{S10}}$; in parallel experiments multiple transformants were obtained for all other mutants. This suggested $\operatorname{arm}^{S 10}$ is dominant lethal. The region deleted in $\mathrm{arm}^{510}$ includes two interesting sequence motifs (Fig. 1A). One is a consensus GSK/Zw3 phosphorylation site (Peifer et al., 1994b). The other is a sequence conserved between IKB and its fly homolog cactus, surrounding the serines whose phosphorylation is thought to regulate IKB ubiquitination and thus control its stability (reviewed by Hochstrasser, 1996).

To test whether $\mathrm{arm}^{S 10}$ is dominant lethal, we expressed an $\mathrm{arm}^{S 10}$ transgene using the inducible GAL-UAS system (Brand and Perrimon, 1993). arm $^{510}$ was cloned downstream of UAS elements regulated by yeast GAL4 and introduced into flies, where it is inactive. arm $^{S 10}$ can be activated in particular tissues at specific times by crossing $\mathrm{arm}^{510}$ lines to lines expressing GAL4 in specific temporal and spatial patterns. We obtained transgenic animals in which either $\mathrm{arm}^{\mathrm{S10}}$, or another mutant, $\operatorname{arm}^{\Delta N}$ (lacking the entire N-terminal domain; Fig. 1A) are under GAL4 regulation. As a control, we placed myc-tagged wild-type Arm under GAL4 control $\left(\mathrm{arm}^{\mathrm{S2}}\right)$. We then used e22c-GAL4 (which expresses GAL4 ubiquitously) to drive essentially ubiquitous expression of our mutant Arm beginning late in embryonic stage 9 .

When activated by e22c-GAL4, $\mathrm{Arm}^{\mathrm{S} 10}, \mathrm{Arm}^{\Delta \mathrm{N}}$ and $\mathrm{Arm}^{\mathrm{S} 2}$ all accumulate to levels roughly similar to that of wild-type Arm (Fig. 1B,C; data not shown); when corrected for gene copy number Arm ${ }^{\mathrm{S} 10}$ levels are approx. 2.4 times those of wildtype Arm (Fig. 1C), while $\mathrm{Arm}^{\Delta \mathrm{N}}$ levels are approx. 1.5 times wild-type Arm $\left(\operatorname{Arm}^{\triangle \mathrm{N} \# 7}\right)$. When analyzed by SDS-PAGE, 

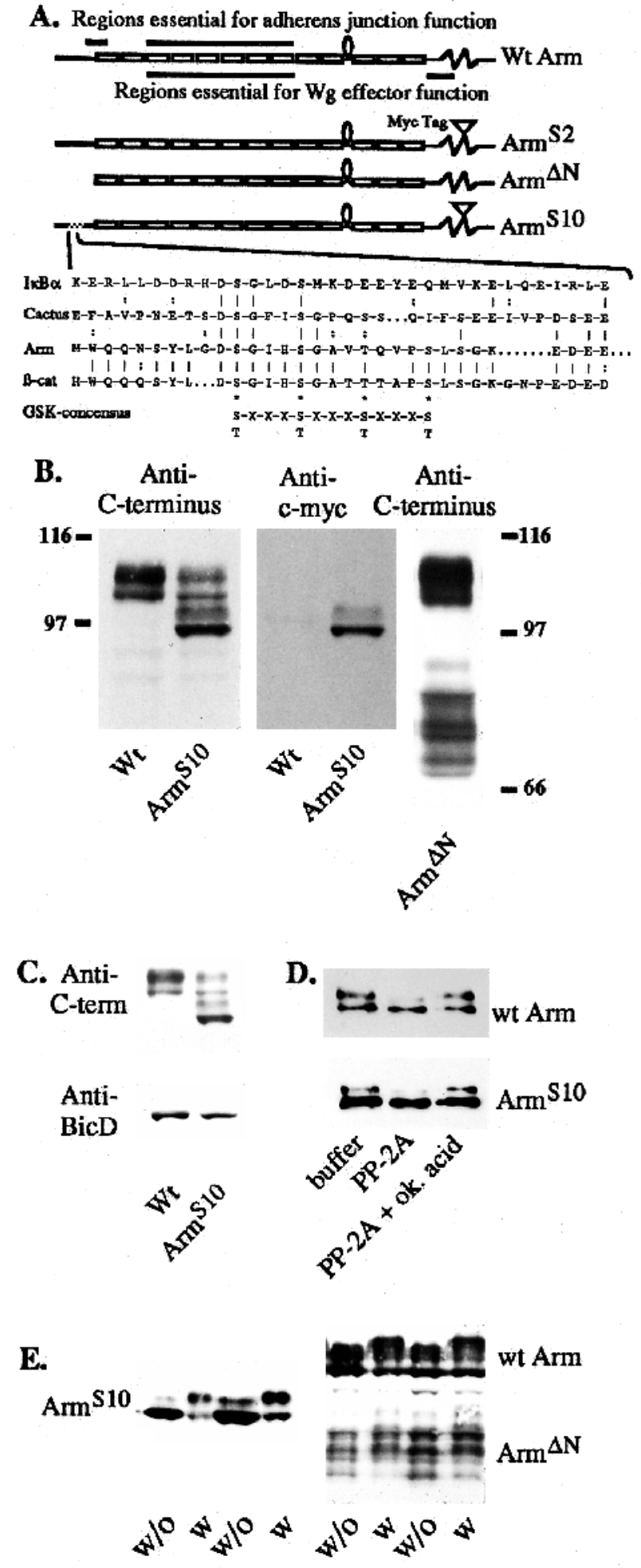

okadaic acid

wild-type Arm accumulates as a set of Ser/Thr phosphorylation variants $\left(105-110 \times 10^{3} M_{\mathrm{r}}\right.$; Peifer et al., 1994b); the most highly phosphorylated isoforms migrate most slowly (Fig. 1B). Both $\mathrm{Arm}^{\mathrm{S} 10}$ and $\mathrm{Arm}^{\Delta \mathrm{N}}$ also have multiple isoforms which result at least in part from Ser/Thr phosphorylation (Fig. 1B). Treatment of Arm ${ }^{\mathrm{S} 10}$ with protein phosphatase $2 \mathrm{~A}$, a Ser/Thrspecific phosphatase, eliminates all but the fastest migrating
Fig. 1. $\mathrm{Arm}^{\mathrm{S} 10}$ and $\mathrm{Arm}^{\Delta \mathrm{N}}$ expression and phosphorylation. (A) Mutant proteins used in this study. Note that $\mathrm{Arm}^{\mathrm{S} 2}$ and $\mathrm{Arm}^{\mathrm{S} 10}$ are tagged with the myc epitope while $\operatorname{Arm}^{\Delta \mathrm{N}}$ is not tagged. The

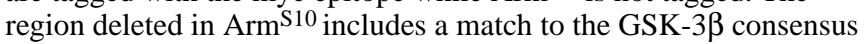
phosphorylation site (Peifer et al., 1994b). It also contains a match to a region conserved between IKB and its fly homolog Cactus, surrounding the serines whose phosphorylation is thought to regulate I $\kappa$ B ubiquitination and thus stability. (B) Both Arm ${ }^{\mathrm{S} 10}$ and $\mathrm{Arm}^{\Delta \mathrm{N}}$ have multiple isoforms. Protein from 0-16 hour old embryos expressing $\mathrm{Arm}^{\mathrm{S} 10}$ or $\mathrm{Arm}^{\Delta \mathrm{N}}$ was analyzed by immunoblotting with anti-ArmCT (recognizing wild-type, $\mathrm{Arm}^{\mathrm{S} 10}$ and $\mathrm{Arm}^{\Delta \mathrm{N}}$ ) or anti-cmyc (recognizing Arm ${ }^{\mathrm{S} 10}$ ). (C) A similar experiment, using ECL detection to allow quantitation; $\mathrm{BicD}$ was used to control loading. $(\mathrm{D}, \mathrm{E}) \mathrm{Arm}^{\mathrm{S} 10}$ is phosphorylated on serine/threonine.

(D) Immunoprecipitates using anti-ArmCT from embryos expressing $\mathrm{Arm}^{\mathrm{S} 10}$ were treated with PP-2A or PP-2A plus its inhibitor okadaic acid. (E) Isolated embryonic cells from $\mathrm{Arm}^{\mathrm{S} 10}$ or $\mathrm{Arm}^{\Delta \mathrm{N}}$ embryos were incubated in D-22 medium with or without the phosphatase inhibitor okadaic acid.

isoform (Fig. 1D), while treatment with okadaic acid, an inhibitor of Ser/Thr-phosphatases, increases the levels of slower migrating $\mathrm{Arm}^{\mathrm{S} 10}$ and $\mathrm{Arm}^{\Delta \mathrm{N}}$ isoforms (Fig. 1E).

Most of the wild-type Arm in an embryo is in adherens junctions, where it is highly phosphorylated; there is relatively little soluble Arm, which is less highly phosphorylated (Peifer et al., 1994b; Fig. 1B). In contrast, the least highly phosphorylated isoforms of $\mathrm{Arm}^{\mathrm{S} 10}$ predominate (Fig. 1B), resembling the pattern of accumulation of wild-type Arm in zw3 mutants (Peifer et al., 1994b). This is not due to ectopic expression; wild-type Arm expressed under GAL4 control is normally phosphorylated (data not shown).

In wild-type embryos, one can follow cell fate choices using cuticular markers; anterior cells within each segment secrete cuticle covered with denticles while posterior cells secrete naked cuticle (Fig. 2A,F). This pattern depends on Wg. A single row of cells underlying the future naked cuticle secrete $\mathrm{Wg}$ and their neighbors respond to this by choosing posterior fates. In embryos mutant for either $w g$ signal or for $\mathrm{arm}$, which is required for signal transduction, all cells assume anterior fates and secrete denticles (Nüsslein-Volhard and Wieschaus, 1980). In the presence of high and ubiquitous levels of $\mathrm{Wg}$, all cells assume posterior fates and secrete naked cuticle (Noordermeer et al., 1992; Sampedro et al., 1993; Yoffe et al., 1995).

Ubiquitous expression of either amino-terminally deleted Arm mutant, $\mathrm{Arm}^{\mathrm{S} 10}$ or $\mathrm{Arm}^{\Delta \mathrm{N}}$, mimics the effects of ubiquitous $\mathrm{Wg}$ expression, producing a phenotype opposite to that of an $\mathrm{arm}$ mutant (Fig. 2). arm $^{S 10}$ lines produce embryos with only naked cuticle or a few denticles (Fig. D,E,G), a phenotype similar to that caused by very high levels of ubiquitous $\mathrm{Wg}$ (Yoffe et al., 1995). Expression of arm $^{S 10}$ using a second ubiquitously expressed driver, 69B-GAL4, had similar effects (Fig. 2). $\operatorname{arm}^{\Delta N}$ lines display partial ablation of the denticle belts, particularly along the ventral midline (Fig. 2B,C); this phenotype overlaps that of $\mathrm{arm}^{S 10}$, but is somewhat weaker and more variable among different transformant lines. The $\mathrm{arm}^{\Delta N}$ phenotype resembles the phenotype seen when $\mathrm{Wg}$ is ubiquitously expressed at lower levels (Noordermeer et al., 1992). As a control we expressed wild-type Arm $\left(\mathrm{arm}^{\mathrm{S}}\right.$; Fig. 1A) ubiquitously, using e22c- or 69B-GAL4. This had no phenotypic consequences; in fact animals carrying $\mathrm{Arm}^{\mathrm{S} 2}$ driven by e22cGAL4 survived to adulthood (data not shown). Further, ubiq- 
uitous expression of wild-type Arm using e22c-GAL4 rescued the embryonic lethality and cuticular phenotype of the zygotic null arm $^{Y D 35}$ (data not shown).

To confirm that the phenotype was caused by the mutant proteins, and to begin to investigate whether their effects are cell autonomous, we expressed them in more restricted patterns. We utilized an engrailed-GAL4 (A. Brand and K. Yoffe, personal communication), which drives transgene expression in posterior cells of each segment; these include both the most posterior cells secreting naked cuticle and the cells secreting the anteriormost row of denticles. When $\mathrm{arm}^{\mathrm{S} 10}$ is expressed in the engrailed domain, the first row of denticles and only the first row of denticles is replaced by naked cuticle (Fig. $2 \mathrm{H}$ versus I), suggesting that $\mathrm{Arm}^{\mathrm{S} 10}$ can act cellautonomously. We also drove $\operatorname{arm}^{\Delta N}$ with hairy-GAL4, which directs expression in even-numbered segments at the cellular blastoderm stage; the resulting embryos had fewer denticles in many even-numbered segments (data not shown).

Both $\mathrm{arm}^{S 10}$ and $\mathrm{arm}^{\Delta N}$ alter the expression of two genes that are known targets of $\mathrm{Wg}$ signaling: engrailed, expressed in two rows of cells immediately posterior to the $w g$-expressing cells (Fig. 3A), and $w g$ itself (Fig. $3 \mathrm{G})$. Ubiquitous expression of either $\operatorname{arm}^{S 10}$ (Fig. 3B) or $\operatorname{arm}^{\Delta N}$ (data not shown) causes a posterior expansion in the domain of en-expressing cells and induces an ectopic stripe of the endogenous Wg (Fig. 3H), as does ubiquitous $\mathrm{Wg}$ expression (Noordermeer et al., 1992; Yoffe et al., 1995).

In a variety of different assays, $\mathrm{arm}^{\Delta N}$ was slightly less potent than $\mathrm{arm}^{S 10}$. This was a difference of degree rather than a qualitative difference. This may be explained by the fact that $\operatorname{arm}^{\Delta N}$ accumulates to slightly lower levels than $\operatorname{arm}^{S 10}$ (Fig. 1B). $\mathrm{arm}^{\Delta N}$ is found as several widely spaced isoforms. The first methionine in $\mathrm{arm}^{\Delta N}$ is not the natural methionine of Arm; this could influence its efficiency of translation, leading to the slightly lower levels. The isoforms may represent starts at other, more internal methionine codons, or they may mean that the protein is somewhat less stable than normal. It is worth noting that $\operatorname{arm}^{\Delta N}$ possesses activated signaling activity despite the fact that it lacks the $\alpha$-catenin binding site (Pai et al., 1996), consistent with independent roles for Arm in signaling and cell adhesion.

\section{The activity of Arm ${ }^{S 10}$ is independent of Wingless signal}

These data suggest that $\mathrm{arm}^{S 10}$ has escaped normal negative regulatory cues and has become constitutively active. If so, it should be independent of endogenous $\mathrm{Wg}$. To test this we expressed $\mathrm{Arm}^{\mathrm{S} 10}$ in a $w g$ mutant where no cell is exposed to $\mathrm{Wg}$. We evaluated cell fate choices by examining both cuticle pattern and $\mathrm{Wg}$ target gene expression. wg null embryos are shorter than wild-type and secrete only denticles with no naked cuticle (Nüsslein-Volhard and Wieschaus, 1980; Fig. 4C). Expression of $\mathrm{Arm}^{\mathrm{S} 10}$ in a $w g$ mutant dramatically alters this (Fig. 4D), producing a cuticle phenotype similar to that of embryos expressing $\mathrm{Arm}^{\mathrm{S} 10}$ in a wild-type background (Fig. 4B). Thus Arm ${ }^{\mathrm{S} 10}$ acts independently of $\mathrm{Wg}$. Once again, $\operatorname{Arm}^{\Delta \mathrm{N}}$ is slightly less active than Arm ${ }^{\mathrm{S} 10} . w g$ mutant embryos expressing $\operatorname{Arm}^{\Delta \mathrm{N}}$ secrete naked cuticle interspersed with denticles on their ventral surface (data not shown), while the dorsal surface is unchanged from the $w g$ mutant phenotype. In contrast, $\mathrm{Arm}^{\mathrm{S} 10}$ expression rescues pattern elements on both
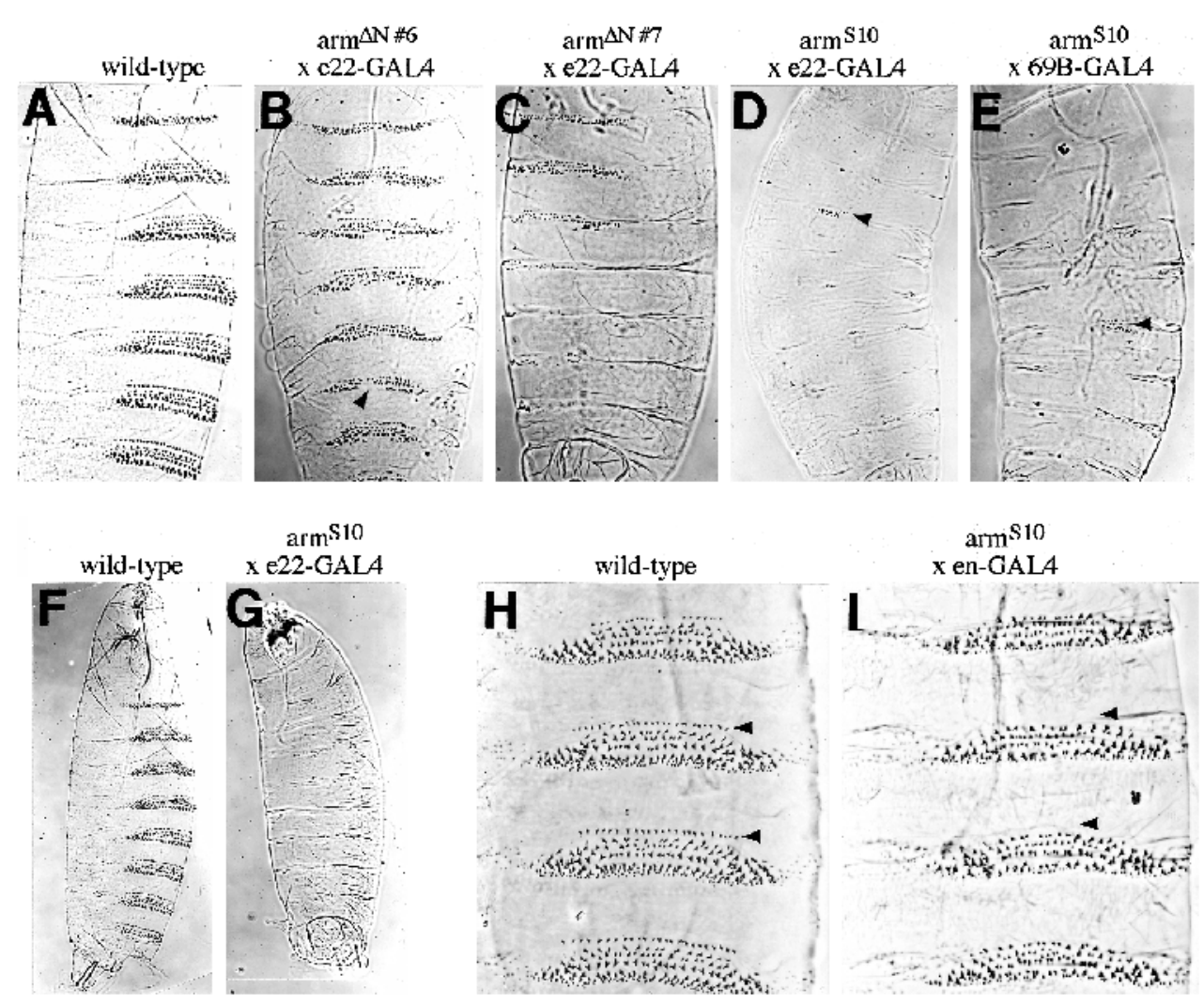

Fig. 2. Ubiquitous expression of $\mathrm{Arm}^{\mathrm{S} 10}$ and $\mathrm{Arm}^{\Delta \mathrm{N}}$ transforms cells to posterior cell fates. Cuticle preparations of wild-type embryos (A,F,H), and of embryos expressing either $\operatorname{Arm}^{\mathrm{S} 10}$ (D,E,G,I) or $\operatorname{Arm}^{\Delta N}(B, C)$ under GAL4 control. e22c-GAL4 and 69B-GAL4 drive essentially ubiquitous expression, while en-GAL4 drives expression specifically in the posterior compartment. (B-G) Ubiquitous expression of either $\mathrm{Arm}^{\mathrm{S} 10}$ or $\mathrm{Arm}^{\Delta \mathrm{N}}$ using e22c-GAL4 drove cells into naked cuticle fates; $\mathrm{Arm}^{\Delta \mathrm{N}}$ lines were weaker in their effects than $\mathrm{Arm}^{\mathrm{S} 10}$ lines. The weakest $\mathrm{Arm}^{\Delta \mathrm{N}}$ lines

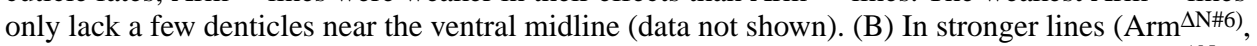
larger regions of naked cuticle appear along the midline (arrowhead). (C) In the strongest $\mathrm{Arm}^{\Delta \mathrm{N}}$ lines $\left(\mathrm{Arm}^{\Delta \mathrm{N} \# 7}\right.$ ), most cells choose naked cuticle fates. (D,E,G) Expression of $\mathrm{Arm}^{\mathrm{S} 10}$ under the control of e22c-GAL4 or 69B-GAL4 caused nearly all cells to choose naked cuticle fates, leaving only a few denticles (arrowheads); unlike zw3 mutants, head structures remain nearly normal. (H,I) When enGAL4 was used to specifically express Arm ${ }^{\mathrm{S} 10}$ in the posterior compartment, which gives rise both to the most posterior cells of the naked cuticle and the anterior row of denticles (arrowheads), the anterior row of denticles was specifically transformed to naked cuticle (arrowheads). 
Fig. 3. Ubiquitous expression of $\mathrm{Arm}^{\mathrm{S} 10}$ and $\mathrm{Arm}^{\Delta \mathrm{N}}$ alters gene expression in a fashion similar to that caused by ubiquitous $\mathrm{Wg}$. Expression of Engrailed (A-F) and Wg (G$\mathrm{H})$ were examined in wildtype or $w g$ mutant embryos with $\mathrm{Arm}^{\mathrm{S} 10}$ or $\mathrm{Arm}^{\Delta \mathrm{N}}$ expressed ubiquitously using e22c-GAL4. (A) Wild-type embryo at stage 9. Engrailed is expressed in two to three rows of cells per segment (arrowheads). (B) In contrast, in embryos expressing $\mathrm{Arm}^{\mathrm{S} 10}$, Engrailed stripes are broadened to three to four rows of cells (arrowheads). (C,F) In $w g$ mutants Engrailed expression begins to decay

during early stage $9(\mathrm{C}$; arrowhead) and is gone from the epidermis by stage 10 (F; remaining expression is in the nervous system). (D,E) Expression of either $\mathrm{Arm}^{\mathrm{S} 10}$ (D) or $\mathrm{Arm}^{\Delta \mathrm{N}}$ (E) in a $w g$ mutant prevents decay of Engrailed stripes; instead stripes are broadened. (G) In wildtype embryos, Wg is secreted by a single row of cells (arrowhead). $(\mathrm{H}) \mathrm{In}$ embryos expressing Arm ${ }^{\mathrm{S} 10}$, an ectopic stripe of $\mathrm{Wg}$ is induced in addition to the endogenous stripe (arrowheads).

the ventral and dorsal surfaces. Using similar GAL4 drivers, ubiquitous $\mathrm{Wg}$ expression produces uniform naked cuticle, as it does in the wild-type background (unpublished data).

In the absence of $\mathrm{Wg}$, Engrailed (En) expression decays between 4 and 5 hours of development (DiNardo et al., 1988; Fig. 3F). Ubiquitous expression of either Arm ${ }^{\mathrm{S} 10}$ (Fig. 3D) or $\operatorname{Arm}^{\Delta \mathrm{N}}$ (Fig. 3E) in a $w g$ mutant causes expansion rather than loss of the En expression domain. Arm ${ }^{\Delta N}$ promotes En expansion only in a ventral domain of cells, while more dorsal parts of the stripe decay (Fig. 3E). Arm ${ }^{\mathrm{S} 10}$ stabilizes and expands the entire En stripe, although the effect is slightly less pronounced in the dorsal region (Fig. 3D). These experiments demonstrate that the effects of $\mathrm{Arm}^{\mathrm{S} 10}$ or $\mathrm{Arm}^{\Delta \mathrm{N}}$ are independent of $\mathrm{Wg}$ ligand and rule out the possibility that the ectopic $\mathrm{Wg}$ expression that they induce is solely responsible for their phenotypic effects.

\section{The activity of ArmS10 is independent of endogenous Armadillo}

The data above can be explained in two distinct ways. Arm ${ }^{\mathrm{S} 10}$ might have escaped normal negative regulation and thus be constitutively active in $\mathrm{Wg}$ signaling. Alternately, $\mathrm{Arm}^{\mathrm{S} 10}$ might have no signaling activity of its own, but might poison the degradation machinery, block degradation of endogenous Arm, and allow it to accumulate and transduce $\mathrm{Wg}$ signal. To distinguish between these two possibilities, we introduced $\mathrm{Arm}^{\mathrm{S} 10}$ into genetic backgrounds depleted for or devoid of wild-type Arm.

We first introduced $\mathrm{Arm}^{\mathrm{S} 10}$ into arm $^{Y D 35}$ embryos, which are zygotically arm null but possess normal levels of maternally contributed Arm. arm ${ }^{Y D 35}$ mutant embryos have a strong segment polarity defect and defects in dorsal closure (Fig. 4E). When $\mathrm{Arm}^{\mathrm{S} 10}$ is expressed in arm $^{Y D 35}$ mutant embryos using e22c-GAL4, the resulting embryos differentiate essentially only naked cuticle (Table 1; Fig. 4F), similar to the phenotype
Table 1. arm $^{S 10}$ activity is independent of endogenous wild-type Arm

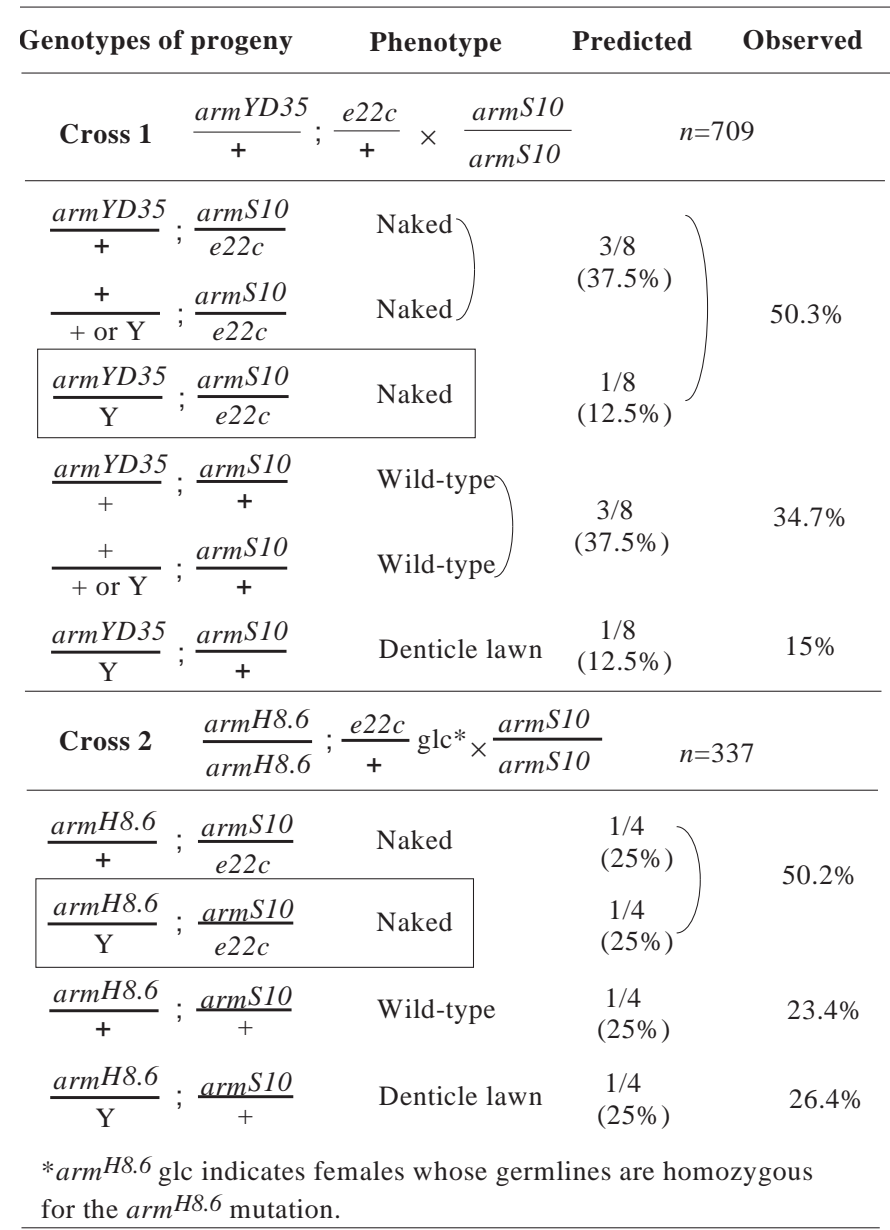


produced by $\mathrm{Arm}^{\mathrm{S} 10}$ expression in wild-type embryos. Thus Arm $^{\mathrm{S} 10}$ acts independently of zygotic Arm. These arm mutant embryos were generally shorter than wild-type embryos expressing $\mathrm{Arm}^{\mathrm{S} 10}$, perhaps due to the relatively late initiation of expression driven by e22c-GAL4 relative to the endogenous arm promotor.

Since the zygotic null still contains maternal wild-type Arm, we also introduced $\mathrm{Arm}^{\mathrm{S} 10}$ into a background in which both the maternal and zygotic Arm contribution were from $\mathrm{arm}^{\mathrm{H} 8.6}$. $\mathrm{Arm}^{\mathrm{H} 8.6}$ protein lacks the $\mathrm{C}$-terminal domain and is nearly null for $\mathrm{Wg}$ signaling (Peifer et al., 1994a). Embryos maternally and zygotically arm $^{H 8.6}$ mutant have a very strong segment polarity phenotype (Fig. 4G), indistinguishable from that of a $w g$ null mutant (Fig. 4C). Expression of $\mathrm{Arm}^{\mathrm{S} 10}$ in such $\mathrm{arm}^{\mathrm{H} 8.6}$ mutant embryos dramatically alters their phenotype; the embryos secrete only naked cuticle (Table 1; Fig. $4 \mathrm{H}$ ), and overlap in phenotype with wild-type embryos expressing Arm $^{\mathrm{S} 10}$.

\section{Arm $\mathrm{S} 10$ is functional in adherens junctions}

Arm has two roles in cells: transducing Wg signal and acting as a linker in adherens junctions. $\mathrm{Arm}^{\mathrm{S} 10}$ binds $\alpha$-catenin and DEcadherin in vivo (Pai et al., 1996), suggesting that it may retain function in junctions. To test this, we introduced $\mathrm{Arm}^{\mathrm{S} 10}$ into an arm $^{X P 33}$ mutant background. arm $^{X P 33}$ protein lacks function in both $\mathrm{Wg}$ signaling and adherens junctions. In embryos maternally and zygotically mutant for $\operatorname{arm}^{X P 33}$, the blastoderm-stage ectodermal epithelium is disrupted and only pieces of cuticle are secreted (Fig. 4I; Cox et al., 1996). If arm $^{X P 33}$ mutant embryos also express $\mathrm{Arm}^{\mathrm{S} 10}$, their cuticle integrity is dramatically improved (Fig. 4J), suggesting that $\mathrm{Arm}^{\mathrm{S} 10}$ retains function in junctions. Furthermore, many embryos make enough cuticle to assay cell fate choices. These embryos resemble wild-type embryos expressing arm $^{S 10}$; essentially all surviving cells secrete naked cuticle (Fig. 4J).

Adherens junctions are required
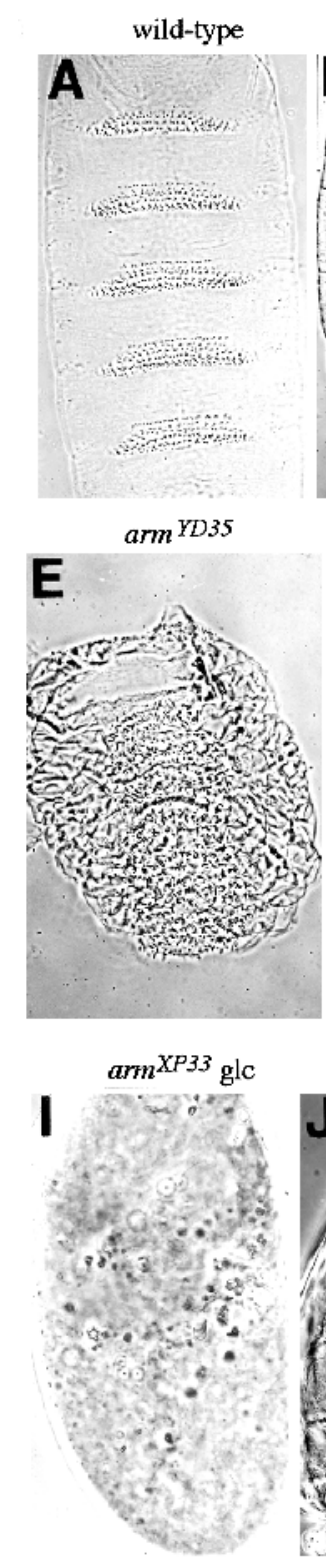

at gastrulation (Cox et al., 1996), while e22c-GAL4 does not activate $\mathrm{Arm}^{\mathrm{S} 10}$ until later. Thus Arm ${ }^{\mathrm{S} 10}$ may not fully rescue junction function because it is expressed too late to do so. To
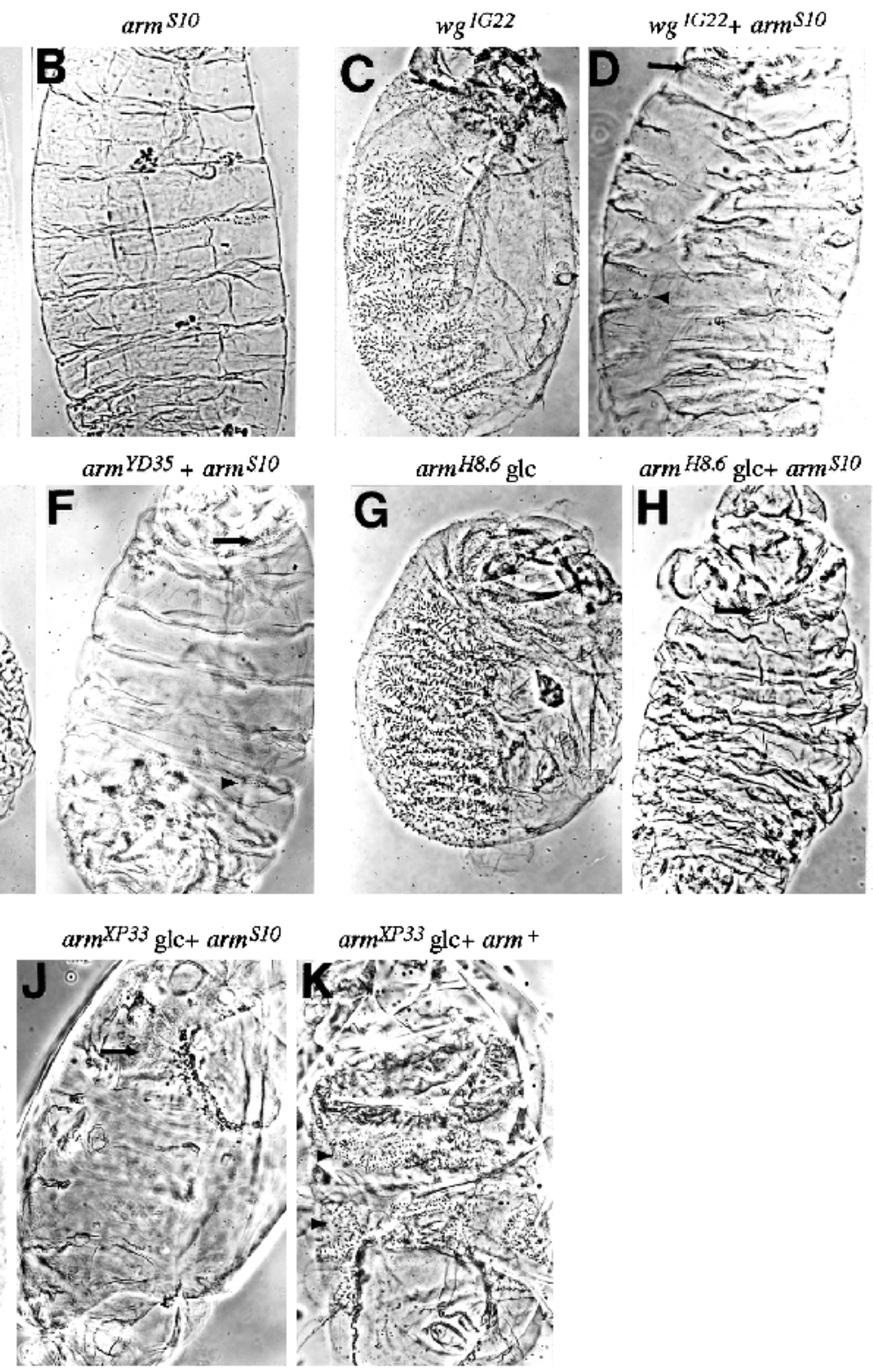

Fig. 4. Arm ${ }^{\mathrm{S} 10}$ is independent of $\mathrm{Wg}$ signal and endogenous Arm activity. Cuticle preparations of wild-type, $w g$ or arm mutant embryos, in some of which $\mathrm{Arm}^{\mathrm{S} 10}$ or $\mathrm{Arm}^{\mathrm{S} 2}$ (wild-type Arm) were expressed ubiquitously using e22c-GAL4. Arrows show the beard of small denticles in the first thoracic segment, confirming that the ventral surface is in focus; arrowheads show residual denticles. (A) Wild-type embryo. (B) Wild-type embryos expressing $\mathrm{Arm}^{\mathrm{S} 10}$. (C) In the $w g$ null $w g^{I G 22}$, all surviving cells choose anterior fates and secrete denticles. (D) Expression of Arm ${ }^{\mathrm{S} 10}$ in $w g$ mutants alters this; virtually all cells choose posterior fates and secrete naked cuticle. (E,G) In the arm zygotic null $\mathrm{arm}^{Y D 35}(\mathrm{E})$, or in embryos expressing only $\mathrm{arm}^{H 8.6}$ mutant protein both maternally and zygotically $\left(\mathrm{G} ; \mathrm{arm}^{\mathrm{H} 8.6} \mathrm{glc}\right)$, all surviving cells choose anterior fates and secrete denticles.

$(\mathrm{F}, \mathrm{H})$ Expression of Arm ${ }^{\mathrm{S} 10}$ alters this, leading most surviving cells to make naked cuticle. In both mutant backgrounds the resulting embryos are shorter than normal; since e22c-GAL4 does not come on strongly till late stage 9 , Arm ${ }^{\mathrm{S} 10}$ may not fully alleviate the consequences of being arm mutant. (I) In embryos expressing only $\mathrm{arm}^{X P 33}$ both maternally and zygotically $\left(\mathrm{arm}^{X P 33} \mathrm{glc}\right)$, adherens junctions disassemble and only scraps of cuticle are made. (J,K) Expression of wild-type Arm $\left(\mathrm{arm}^{+}\right)$via e22c-GAL4 in this background substantially restores cuticle integrity (K), as does expression of $\mathrm{Arm}^{\mathrm{S} 10}(\mathrm{~J})$. However, while expression of wild-type Arm restores both denticles and naked cuticle, the cuticle restored by $\mathrm{Arm}^{\mathrm{S} 10}$ is essentially completely naked. 


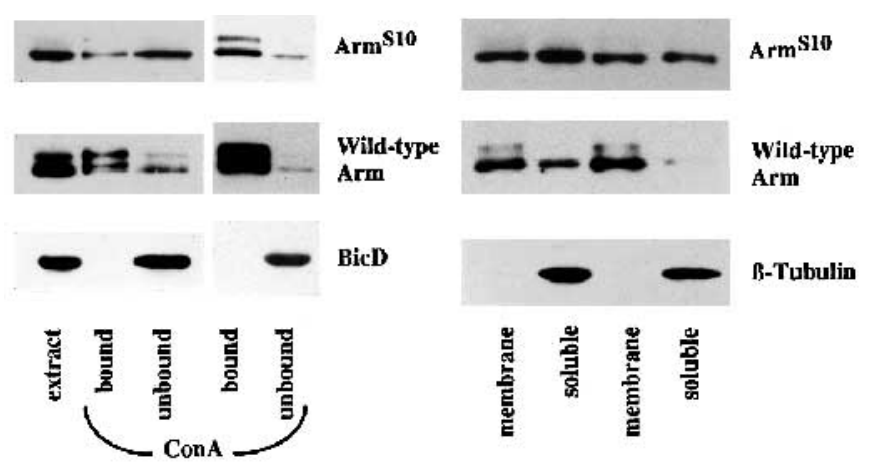

Fig. 5. Arm ${ }^{\mathrm{S} 10}$ accumulates at high levels outside adherens junctions. Embryos expressing both wild-type Arm and $\mathrm{Arm}^{\mathrm{S} 10}$ were fractionated using either Con A-Sepharose to isolate cadherin-bound Arm (left panel) or by centrifugation to separate a membrane fraction (which also contains nuclei) and a soluble fraction (right panel). Fractions were analyzed by SDS-PAGE and sequential immunoblotting with anti-c-myc (recognizing Arm ${ }^{\mathrm{S} 10}$ ), anti-Arm N terminus (recognizing wild-type Arm), or anti-BicD or anti- $\beta$-tubulin as fractionation controls. (Left panel) In the lanes at the left, approximately equal portions of the Con A-bound and unbound fractions were loaded. In the lanes at the right about 3 times as much of the Con A-bound fraction was loaded. While only about onefourth of wild-type Arm is outside adherens junctions, nine-tenths of the $\mathrm{Arm}^{\mathrm{S} 10}$ is in the unbound fraction. (Right panel) $0-16$ hour old (left lanes) or 4-8 hour old embryos (right lanes) were separated into P100 (membrane) and S100 (soluble) fractions. In both cases, about 5 times as much of the membrane fraction is loaded. Relatively little wild-type Arm is in the soluble fraction; in contrast the majority of $\mathrm{Arm}^{\mathrm{S} 10}$ is in the soluble fraction.

test this we expressed wild-type Arm $\left(\mathrm{Arm}^{\mathrm{S} 2}\right)$ using the same GAL4 driver in the same mutant background. Arm ${ }^{\mathrm{S} 2}$ rescues arm $^{X P 33}$ cuticle integrity to the same degree as Arm ${ }^{\mathrm{S} 10}$, however, unlike $\mathrm{Arm}^{\mathrm{S} 10}, \mathrm{Arm}^{\mathrm{S} 2}$ allows some cells to secrete denticles (Fig. 4K). Thus Arm ${ }^{\mathrm{S} 10}$ functions normally at adherens junctions even though it is constitutively active for $\mathrm{Wg}$ signaling. Furthermore, this confirms that Arm ${ }^{\mathrm{S} 10}$ activity does not require wild-type Arm, since $\mathrm{arm}^{X P 33}$ is completely null for $\mathrm{Wg}$ signaling.

\section{Arm ${ }^{\mathbf{S 1 0}}$ is stabilized outside adherens junctions}

If $\mathrm{Arm}^{\mathrm{S} 10}$ has escaped negative regulation, it should accumulate stably outside adherens junctions in all cells, regardless of whether they have been exposed to $\mathrm{Wg}$. To test this, we isolated adherens junction complexes using the lectin Con ASepharose, which binds $\alpha$-linked mannose residues on DEcadherin. We then compared the amount of both $\mathrm{Arm}^{\mathrm{S} 10}$ and wild-type Arm in the bound (junctional) and the unbound (nonjunctional) fractions (Fig. 5, left panels), by immunoblotting with anti-c-myc, which specifically recognizes $\mathrm{Arm}^{\mathrm{S} 10}$, and with anti-Arm 7A1, which specifically recognizes wild-type Arm. Most wild-type Arm is in adherens junctions; in contrast $\mathrm{Arm}^{\mathrm{S} 10}$ accumulates to high levels outside adherens junctions. We also fractionated embryos into membrane and soluble fractions (Fig. 5, right panels). Most wild-type Arm is in the membrane fraction; in contrast, levels of $\mathrm{Arm}^{\mathrm{S} 10}$ are relatively high in the soluble fraction, especially at embryonic stages 911 (4-8 hours) when $\mathrm{Wg}$ signaling is maximal. Hence, while $\mathrm{Arm}^{\mathrm{S} 10}$ associates with adherens junctions, it also accumulates to high levels in the cytoplasm, unlike wild-type Arm. This difference is not simply due to the myc-tag, as it has been previously demonstrated that $\mathrm{Arm}^{\mathrm{S} 2}$ and a subset of other myctagged mutant Arm proteins have subcellular localizations indistinguishable from that of wild-type Arm (Orsulic and Peifer, 1996b).

To confirm these biochemical data, we examined Arm ${ }^{S 10}$ localization in situ (Fig. 6). Wild-type Arm accumulates in adherens junctions of all cells, thus outlining cells of the embryonic epidermis (Fig. 6A,D). In cells not receiving $\mathrm{Wg}$ signal there is little Arm in the cytoplasm or nucleus. In contrast, in cells receiving Wg signal, Arm accumulates to high levels in the cytoplasm and nucleus (Orsulic and Peifer,

Fig. 6. Arm $\mathrm{S}^{\mathrm{S} 10}$ accumulates at high levels in the cytoplasm and nucleus of all cells, regardless of whether they have received Wg signal. Embryos were prepared for immunofluorescence, to visualize either wild-type Arm (A and C, anti-Arm N2; D, anti-CT), $\operatorname{Arm}^{\mathrm{S} 10}$ (B, anti-c-myc), or both (E, anti-CT). (A,D) Wild-type Arm accumulates in adherens junctions of all cells, and also accumulates in the cytoplasm and nucleus of segmentally reiterated rows of cells which receive $\mathrm{Wg}$ signal. (B,E) Arm ${ }^{\mathrm{S} 10}$ accumulates in the membrane, cytoplasm and nucleus of all cells, regardless of whether they received $\mathrm{Wg}$ signal. Similar uniform staining of the entire cell was seen in a variety of focal planes, suggesting that $\mathrm{Arm}^{\mathrm{S} 10}$ accumulates in both the cytoplasm and nuclei, as does wild-type Arm in cells receiving $\mathrm{Wg}$ signal. The inset in $\mathrm{E}$ shows an embryo from the same field which is

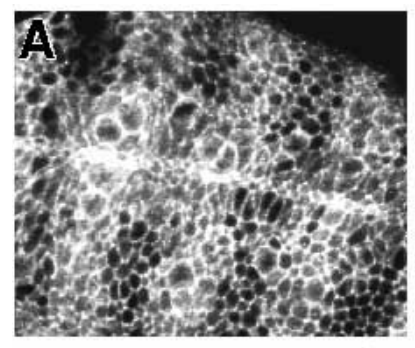
Wild-type Arm

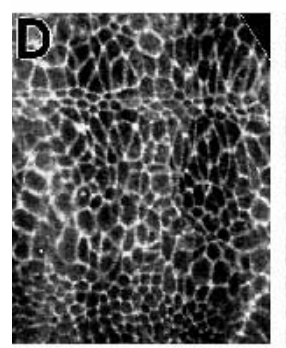

Wild-typc Atm

$\mathrm{Arm}^{\mathrm{S10}}$

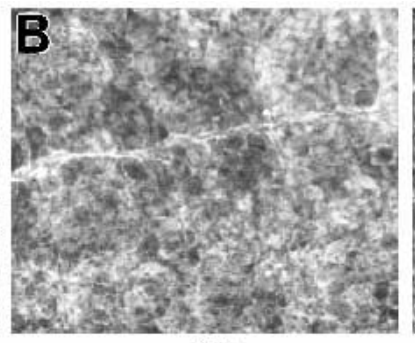

Arm 510

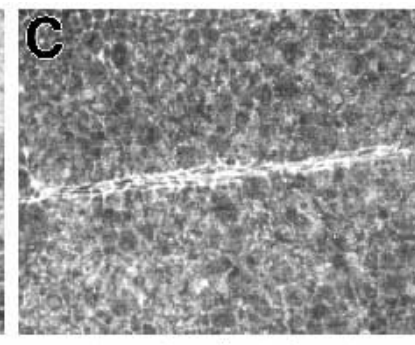

Wild-type Arm in $z w 3$ mutant

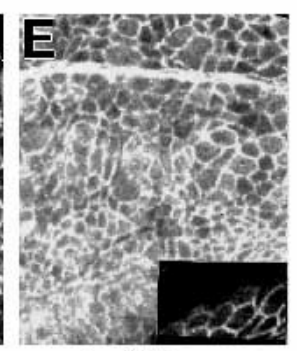
just gastrulating and thus is prior to the onset of expression of $\mathrm{Arm}^{\mathrm{S} 10}$. (C) In a zw3 mutant embryo, wild-type Arm accumulates in the membrane, cytoplasm and nucleus of all cells, regardless of whether they received $\mathrm{Wg}$ signal. 


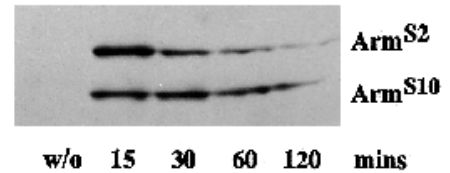

Fig. 7. $\mathrm{Arm}^{\mathrm{S} 10}$ is more stable than wild-type Arm. 0-16 hour old

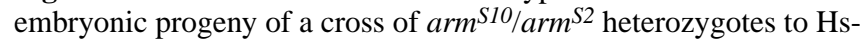
GAL4 homozygotes were collected. A portion were ground in sample buffer without heat shock. The rest were heat-shocked for 50 minutes. Embryos were collected at 15, 30, 60 and 120 minutes after heat shock and ground in sample buffer. Arm ${ }^{\mathrm{S} 2}$ and $\mathrm{Arm}^{\mathrm{S} 10}$ were simultaneously detected by immunoblotting with anti-c-myc. In this experiment, the half-life of $\mathrm{Arm}^{\mathrm{S} 2}$ was 12 minutes while that of Arm $^{\mathrm{S} 10}$ was 67 minutes.

1996b). In zw3 mutants (Fig. 6C), however, Arm accumulates at high levels in the cytoplasm and nucleus of all cells, regardless of whether they were exposed to Wg (Peifer et al., 1994a; Siegfried et al., 1994).

We first localized $\mathrm{Arm}^{\mathrm{S} 10}$ (expressed in a wild-type background under the control of the ubiquitously expressed e22cGAL4) using anti-c-myc antibody, which only recognizes $\mathrm{Arm}^{\mathrm{S} 10}$ and not wild-type Arm. The distribution of $\mathrm{Arm}^{\mathrm{S} 10}$ is strikingly different from that of wild-type Arm; $\mathrm{Arm}^{\mathrm{S} 10}$ accumulates to high levels in the cytoplasm and nucleus of all cells (Fig. 6B). This distribution resembles in detail the distribution of wild-type Arm in a zw3 mutant (Fig. 6C). We observed an essentially identical localization of $\mathrm{Arm}^{\mathrm{S} 10}$ using an antibody directed against the $\mathrm{C}$ terminus of Arm, which thus recognizes both wild-type Arm and $\mathrm{Arm}^{\mathrm{S} 10}$. In blastoderm and gastrulating embryos, prior to the onset of GAL4 and thus Arm ${ }^{\mathrm{S} 10}$ expression, the antibody showed a distribution of Arm indistinguishable from that observed in wild-type embryos (Fig. $6 \mathrm{E}$, inset; data not shown). In late stage 9 , however, after the onset of $\mathrm{Arm}^{\mathrm{S} 10}$ expression, Arm accumulates both at cell junctions and within the nucleus and cytoplasm of all cells (Fig. 6E), regardless of their position within the segment. In these two experiments we used two different ubiquitous GAL4 drivers, confirming that the observed pattern of intracellular accumulation is not specific to a particular GAL4 driver.

\section{$\mathrm{Arm}^{\mathrm{S10}}$ is more stable than wild-type Arm}

The level of Arm protein is regulated via effects on its half life (van Leeuwen et al., 1994). Since Arm ${ }^{\mathrm{S} 10}$ accumulates at high levels in the cytoplasm and nucleus, as wild-type Arm does in a $z w 3$ mutant, we examined the protein stability of $\mathrm{Arm}^{\mathrm{S} 10}$. The large maternal pool prevented us from achieving sufficient labeling to measure protein stability by pulse-labeling embryos with ${ }^{[35}$ S $]$ methionine. We thus took an alternative approach, using Hs-GAL4 to briefly activate transgene expression. To compare the half-life of $\mathrm{Arm}^{\mathrm{S} 10}$ to that of wild-type Arm under exactly the same conditions, we crossed flies heterozygous for $\mathrm{Arm}^{\mathrm{S} 10} / \mathrm{Arm}^{\mathrm{S} 2}$ to flies homozygous for Hs-GAL4. Of the resulting progeny, half express $\mathrm{Arm}^{\mathrm{S} 10}$ and half $\mathrm{Arm}^{\mathrm{S} 2}$. Embryos were briefly heat-shocked to initiate expression of both transgenes, and at various times embryos were collected, extracted, and analyzed by immunoblotting. We found that $\mathrm{Arm}^{\mathrm{S} 10}$ had a longer half-life than $\mathrm{Arm}^{\mathrm{S} 2}$ (Fig. 7). In the experiment shown the half-life of wild-type Arm was 12 minutes while that of $\mathrm{Arm}^{\mathrm{S} 10}$ was 67 minutes. A low level of both proteins remained even at the longest timepoint; this pool of
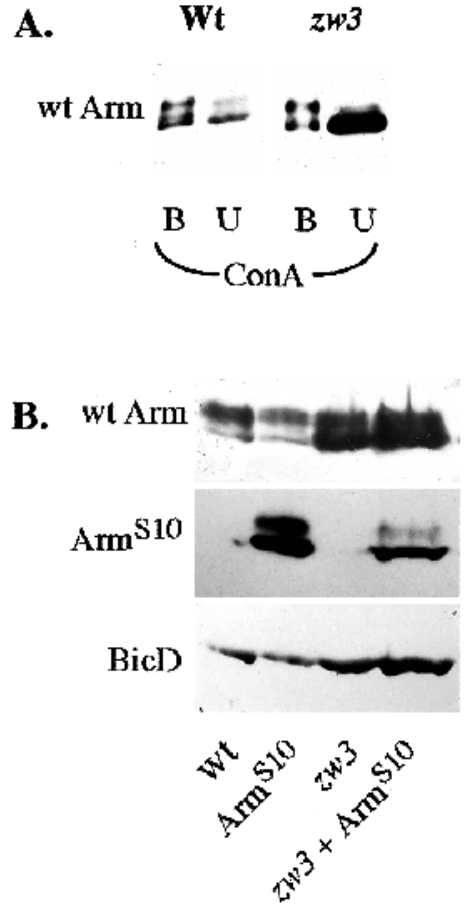

C. Wt zw3 $\stackrel{z w 3+}{\mathrm{Arm}^{\mathrm{S} 10} \mathrm{Arm}^{\mathrm{S} 10}}$
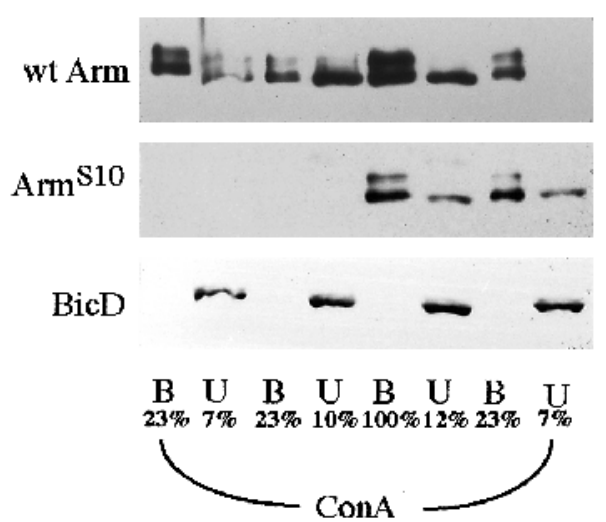

Fig. 8. Zw3 kinase does not regulate the stability of Arm ${ }^{\mathrm{S} 10}$. (A) Junctional wild-type Arm is phosphorylated normally in a $z w 3$ mutant. 4-9 hour old wild-type or zw3 mutant embryos were separated into Con A-bound (adherens junction-associated) and unbound (nonjunctional) fractions. The expression level and phosphorylation status of junctional wild-type Arm is not altered in zw3 mutants, while the soluble, hypophosphorylated isoform of Arm is stabilized. (B) While the total level of wild-type Arm is increased in a zw3 mutant, the total level of $\mathrm{Arm}^{\mathrm{S} 10}$ is reduced. 2-10 hour old embryos from wild-type, $z w 3, \mathrm{Arm}^{\mathrm{S} 10}$ in wild-type, and $\mathrm{Arm}^{\mathrm{S} 10}$ in zw3 mutant backgrounds were analyzed by immunoblotting with antibodies recognizing wild-type Arm (7A1), $\mathrm{Arm}^{\mathrm{S} 10}$ (c-myc) and $\mathrm{BicD}$ (as a loading control). (C) The level of nonjunctional $\mathrm{Arm}^{\mathrm{S} 10}$ is unaltered in a $z w 3$ mutant, while the level of junctional $\mathrm{Arm}^{\mathrm{S} 10}$ is reduced. 2-15 hour old embryos of the genotypes in the previous panel were fractionated into Con A-bound (B; junctional) and unbound (U; non-junctional) fractions. Percentages indicate the percentage of the total sample loaded. Note that substantially more of the bound fraction was loaded in the sample to analyze Arm $\mathrm{S10}$ accumulation in a $z w 3$ mutant background. 
long-lived protein may be junctional. Longer heat-shocks resulted in less pronounced differences; this may be due to production of a large enough pool of GAL4 to produce high levels of synthesis throughout the experiment.

\section{The stability of non-junctional Arm $^{\mathrm{S} 10}$ is not regulated by Zw3}

We previously suggested that Zw3 kinase is required for Arm phosphorylation, and that phosphorylation levels affect Arm protein stability (Peifer et al., 1994b). An alternate hypothesis is also tenable, however. The kinase that phosphorylates Arm may reside in adherens junctions. If so, in a wild-type embryo overall levels of Arm phosphorylation would be high, as most Arm is assembled in junctions and little is in the cytoplasm or nucleus. If the primary effect of $z w 3$ mutations is to increase Arm stability outside junctions, this would result in an apparent net reduction in Arm phosphorylation, due to an increase in the abundance of the hypophosphorylated form found outside junctions.

To distinguish between these alternatives, we used Con ASepharose to separate wild-type Arm in adherens junctions from non-junctional Arm in a zw3 mutant background. Even though overall levels of Arm phosphorylation are low in the zw3 mutant because the less phosphorylated isoform accumulates highly outside junctions (Fig. 8A), junctional Arm remains highly phosphorylated. Consistent with this, the most highly phosphorylated isoforms of $\mathrm{Arm}^{\mathrm{S} 10}$ also accumulate preferentially in junctions (Fig. 5). Together, these data support the hypothesis that the kinase producing the Ser/Thr phosphorylation isoforms of both wild-type Arm and of $\mathrm{Arm}^{\mathrm{S} 10}$ is junctional, and demonstrate that junctional Arm can be phosphorylated by a kinase other than that encoded by $z w 3$.

The phenotypic analyses described above are consistent with the idea that $\mathrm{Arm}^{\mathrm{S} 10}$ has escaped from normal regulation by Zw3. We tested this biochemically by expressing $\operatorname{Arm}^{\mathrm{S} 10}$ in a zw3 mutant background. Both expression of $\mathrm{Arm}^{\mathrm{S} 10}$ and maternal absence of $z w 3$ produce embryos with entirely naked cuticles; not surprisingly, we could not clearly distinguish two classes of cuticles representing zw3 mutant embryos that did or did not express $\mathrm{Arm}^{\mathrm{S} 10}$ (data not shown).

We examined the effect of zw3 mutations on levels of intracellular $\mathrm{Arm}^{\mathrm{S} 10}$. While loss of $z w 3$ substantially increases the total level of wild-type Arm (Peifer et al., 1994a,b; Fig. 8B), the total level of ArmS10 actually decreases in $z w 3$ mutants (Fig. 8B). The effects of $z w 3$ mutations on levels of wild-type Arm result from the stabilization of less phosphorylated wild-type Arm outside junctions in $z w 3$ mutants (Peifer et al. 1994a,b; Fig 8A,C). In contrast, zw3 mutations do not alter the already high levels of $\mathrm{Arm}^{\mathrm{S} 10}$ outside junctions (Fig. 8C). Instead, zw3 mutations decrease the amount of junctional $\mathrm{Arm}^{\mathrm{S} 10}$. This latter effect may be a secondary consequence of the stabilization of wild-type Arm; the substantially increased pool of wild-type Arm may effectively compete for binding to cadherin, displacing some of the junctional $\mathrm{Arm}^{\mathrm{S} 10}$. Thus Zw3 kinase does not regulate the stability of $\mathrm{Arm}^{\mathrm{S} 10}$ outside junctions; in fact $\mathrm{Arm}^{\mathrm{S} 10}$ behaves in a wild-type background in a fashion very similar to wild-type Arm in a $z w 3$ mutant background, consistent with the idea that $\mathrm{Arm}^{\mathrm{S} 10}$ has escaped from regulation by $\mathrm{Zw} 3$.

\section{DISCUSSION}

\section{A region of Armadillo negatively regulates Armadillo's activity in Wg signal transduction}

Molecular and genetic analyses in Drosophila have suggested that Zw3 kinase is a ubiquitous negative regulator of $\mathrm{Wg}$ signal transduction; in cells exposed to $\mathrm{Wg}$ this negative regulation is antagonized, allowing Arm to act as an effector. We identified a region of Arm essential for this negative regulation. Removal of the entire $\left(\mathrm{Arm}^{\Delta \mathrm{N}}\right)$ or the central part $\left(\mathrm{Arm}^{\mathrm{S} 10}\right)$ of the $\mathrm{N}$ terminus of Arm leads to its constitutive activation in $\mathrm{Wg}$ signal transduction. We confirmed this by showing that the activity of $\mathrm{Arm}^{\mathrm{S} 10}$ is independent of $\mathrm{Wg}$ signal, as assayed both by its effects on embryonic pattern and its ability to activate the downstream genes engrailed and $w g$. Consistent with this, mutations in Armadillo's partner in the signal transduction pathway, the transcription factor $d T C F$, block the action of $\mathrm{Arm}^{\mathrm{S} 10}$ in double mutants (van de Wetering et al., 1997; Brunner et al., 1997). As part of their examination of $\mathrm{Wg}$ signaling in imaginal discs, Zecca et al. (1996) recently demonstrated that a mutant version of Arm similar to our $\operatorname{Arm}^{\Delta N}$ results in constitutive signaling activity in embryos, as assayed by cuticle pattern, and in imaginal discs, as assayed by molecular markers.

\section{Arm $^{\mathbf{S 1 0}}$ is capable of transducing $\mathbf{W g}$ signal}

In Xenopus overexpression of $\mathrm{N}$-terminal $\beta$-catenin mutants activates an apparent Wnt signaling pathway (Yost et al., 1996). Since Arm/ $\beta$-catenin degradation is promoted by Zw3/GSK kinase and the tumor suppressor APC, possibly as part of a larger complex (Rubinfeld et al., 1996), there are two mechanisms by which $N$-terminal mutants of Arm/ $\beta$-catenin could affect signaling. First, they could form non-productive complexes with the degradation machinery, allowing wild-type Arm $/ \beta$-catenin to accumulate to high levels and thus mediate signaling. Alternately, these mutants could both evade degradation and act in signaling themselves. We discriminated between these two models, using a genetic approach. Arm $\mathrm{S} 10$ itself transduces $\mathrm{Wg}$ signal, even in the absence of wild-type Arm.

This does not, however, rule out the possibility that overexpression of mutant Arm/ $\beta$-catenin might block degradation. Such dominant inhibition of degradation might explain an apparent discrepancy in previous work. In flies, the Arm C terminus is essential for $\mathrm{Wg}$ signal transduction (Peifer et al., 1994a); it acts as a transcriptional activation domain in a complex with the dTCF DNA-binding protein (van de Wetering et al., 1997). In contrast, in Xenopus, over-expression of the $\beta$-catenin Arm repeats without the $C$ terminus activates signaling (Funayama et al., 1995). This mutant $\beta$-catenin should bind APC, and thus might block the Xenopus degradation machinery, allowing wild-type $\beta$-catenin to accumulate for signaling. This can now be tested by expressing analogous mutants in flies in the absence of endogenous Arm.

\section{$\mathrm{Arm}^{\mathrm{S} 10}$ has escaped normal regulation by Zw3 kinase}

The constitutive activity of N-terminal arm mutants is not due to overexpression. Moderate over-expression of wild-type Arm by gene duplication (Orsulic and Peifer, 1996b) or using GAL4 (see above) has no effect on development; presumably the 
endogenous degradation system can degrade the excess protein. Further, the accumulation level of $\operatorname{Arm}^{\Delta N}$ is almost identical to that of wild-type Arm (Fig. 1).

Instead, our data strongly suggest that $\operatorname{Arm}^{\mathrm{S} 10}$ and $\mathrm{Arm}^{\Delta \mathrm{N}}$ are not down-regulated by Zw3. Normally Zw3 destabilizes soluble Arm, allowing Arm to accumulate only in adherens junctions (Peifer et al., 1994a). In contrast, Arm ${ }^{\mathrm{S} 10}$ accumulates at high levels outside junctions even in the presence of wild-type Zw3 kinase (Figs 5, 6); this extrajunctional accumulation of $\mathrm{Arm}^{\mathrm{S} 10}$ is not altered by $z w 3$ mutations (Fig. 8), and the half-life of $\mathrm{Arm}^{\mathrm{S} 10}$ is longer than that of wild-type Arm (Fig. 7).

Our data are largely consistent with those on $\beta$-catenin. Nterminally mutant $\beta$-catenin has a longer half-life in Xenopus embryos, accumulates outside adherens junctions, and is more potent in Wnt signaling (Yost et al., 1996). Likewise, N-terminally deleted or point mutated $\beta$-catenin is stabilized in the soluble pool in mammalian epithelial cells (Munemitsu et al., 1996; Rubinfeld et al., 1997), and activates transcription via association with Lef-1 or TCF-4 (Rubinfeld et al., 1997; Morin et al., 1997; Korinek et al., 1997). The increased stability of $\mathrm{Arm}^{\mathrm{S} 10}$ in Drosophila embryos was not as dramatic as that observed in vertebrates. This may result from the fact that we measured protein stability in intact, late-stage embryos; many cells are exposed to $\mathrm{Wg}$, stabilizing wild-type Arm and minimizing differences with Arm ${ }^{\mathrm{S} 10}$. In addition, our assay measured protein half-life indirectly, potentially minimizing stability differences.

\section{Armadillo and APC; possible targets of Zw3 kinase}

Two facts are clear. First, the Ser/Thr kinase Zw3/GSK is an essential negative regulator of Arm/ $\beta$-catenin's role in signaling (Siegfried et al., 1994; Peifer et al., 1994a,b; Pierce and Kimelman, 1995; Yost et al., 1996). Second, the Arm/ $\beta$ catenin $\mathrm{N}$ terminus is essential for this negative regulation (our data; Yost et al., 1996; Munemitsu et al., 1996); point mutants in a putative phosphorylation site within this region have similar effects (Yost et al., 1996; Morin et al, 1997; Rubinfeld et al., 1997), strongly suggesting that phosphorylation is the means of regulation.

These data suggested a simple model, whereby Zw3/GSK kinase negatively regulates Arm/ $\beta$-catenin by directly phosphorylating it on a site within the $\mathrm{N}$ terminus. Consistent with this, Arm is a phosphoprotein, and its average level of phosphorylation is greatly reduced in zw3 mutants (Peifer et al., 1994b). Zw3/GSK consensus phosphorylation sites are found in Arm's N- and C-terminal domains (Peifer et al., 1994b). While the C-terminal site is not essential for function (Orsulic and Peifer, 1996b), the N-terminal site is within the Arm $\mathrm{S10}$ deletion. The equivalent $\mathrm{N}$-terminal site within $\beta$-catenin is a target for GSK in vitro; point mutations in this site block in vitro phosphorylation and also activate the protein in signaling (Yost et al., 1996).

In our hands, however, the equivalent site within Arm is not avidly phosphorylated by GSK, even if the site is pre-phosphorylated by casein kinase II (unpublished data); others have suggested that the site within $\beta$-catenin is also not a favored site for GSK (Stambolic et al., 1996). Thus a second hypothesis must be considered. An alternative direct target of Zw3/GSK is the tumor suppressor APC, which is readily phos- phorylated by GSK. This phosphorylation regulates APC binding to $\beta$-catenin (Rubinfeld et al., 1996), reducing $\beta$ catenin stability (Munemitsu et al., 1995). In this model a different kinase might phosphorylate the $\mathrm{N}$-terminal site within Arm $/ \beta$-catenin. In support of this alternative model, we found that our earlier data documenting differences in Arm phosphorylation between wild-type and zw3 mutants are at least in part an indirect result of alterations of the ratio of junctional to soluble Arm. Further, junctional Arm is equally highly phosphorylated in both wild-type and zw3 mutant embryos. Thus Zw3 kinase is not required for phosphorylation of Arm in adherens junctions, suggesting that this phosphorylation is mediated by other kinases. The effect of Zw3 inactivation on Arm phosphorylation may be solely due to its effects on the stability of soluble Arm.

Arm phosphorylation is thus more complex than envisioned in the simplest model. Junctional kinases apparently phosphorylate Arm. The visible isoforms of Arm are not solely produced by Zw3. Further, there are sites for Arm phosphorylation outside of the central $\mathrm{N}$ terminus, as $\mathrm{Arm}^{\mathrm{S} 10}$ has nearly as many phosphorylated isoforms as wild-type Arm. These data are consistent with a model in which Arm is an indirect target of Zw3. They also are consistent with the possibility that Zw3 phosphorylates Arm, if certain other conditions are met. For example, phosphorylation might occur in an APC:Arm:Zw3 complex, in which the half-life of phosphorylated Arm is extremely short. Alternately, Zw3 may phosphorylate Arm at sites which do not alter its mobility. More complex models exist in which APC and Arm/ $\beta$-catenin are sequential targets of Zw3/GSK.

The mechanism by which Arm/ $\beta$-catenin is destabilized also remains a mystery. The ubiquitin/proteasome system regulates the stability of a wide variety of soluble as well as transmembrane proteins. One of the best studied examples is $I \kappa B$, the inhibitor of NF- $\kappa \mathrm{B}$ nuclear entry. Ubiquitination and destruction of $\mathrm{I} \kappa \mathrm{B}$ is somehow associated with its phosphorylation (reviewed by Hochstrasser, 1996), though which event is primary remains uncertain. It is interesting to note that there is a weak match between the region surrounding the known sites of $\mathrm{I} \kappa \mathrm{B}$ phosphorylation and the region of Arm deleted in $\mathrm{Arm}^{\mathrm{S} 10}$ (Fig. 1A); it is thus possible that Arm degradation is triggered by phosphorylation by the mysterious high molecular weight kinase thought to phosphorylate IKB, with Zw3 acting only on APC.

The tools to analyse APC's role of Wg/Wnt signaling in vivo are now becoming available. Hayashi et al. (1997) recently cloned a fly relative of APC. Surprisingly, embryos zygotically null for APC have normal cuticle pattern and normal patterns of Arm accumulation (Hayashi et al., 1997); it will be very interesting to learn whether removal of maternal APC has any phenotypic consequences for embryogenesis. Surprisingly, injection of mRNA encoding full-length APC into Xenopus embryos led to activation, rather than negative regulation of $\beta$ catenin's role in signaling (Vleminckx et al., 1997). These data suggest that we still have much to learn about APC's roles in vivo.

These data, along with those from the vertebrate system, set the stage for a detailed examination of the mechanism by which Arm is regulated. This will reveal how reception of $\mathrm{Wg} / \mathrm{Wnt}$ signals directs key events of normal development, and will also help clarify a potential role for Arm activation in tumorigen- 
esis. Levels of soluble $\beta$-catenin are highly elevated in colon tumors, due to loss of wild-type APC (Munemitsu et al., 1995). Further, mutations in the $\mathrm{N}$ terminus of $\beta$-catenin very similar to those in $\mathrm{Arm}^{\mathrm{S} 10}$ are oncogenic in culture (Whitehead et al., 1995) and appear to play a causal role in a variety of different tumors (Kawanishi et al., 1995; Robbins et al., 1996; Morin et al, 1997; Rubinfeld et al., 1997). These mutations dramatically raise the levels of non-junctional $\beta$-catenin, driving the formation of complexes with members of the TCF/LEF family and re-programming gene expression (Korinek et al, 1997; Rubinfeld et al., 1997). Together these data suggest that mutational activation of $\beta$-catenin may be one way in which cells escape normal growth controls.

We thank A. Brand, S. DiNardo, N. Perrimon and the Bloomington Drosophila Stock Center for fly stocks and vector DNAs, J. Shields and C. Brown for lab management, D. Sweeton and E. Wieschaus for the picture of Arm in zw3, S. Whitfield for photography, and C. McCormick, L. Rose, A. Rougvie, lab members and the anonymous reviewers for comments on the manuscript. This work was supported by grants from NIH (GM47857) and the Searle Scholars Program to M.P., and by Basil O'Connor Starter Scholar Research Award FY95-1107 from the March of Dimes Birth Defects Foundation to A. B.

\section{REFERENCES}

Behrens, J., Kries, J. P. v., Kühl, M., Bruhn, L., Wedlich, D., Grosschedl, R. and Birchmeier, W. (1996). Functional interaction of beta-catenin with the transcription factor LEF-1. Nature 382, 638-642.

Bhanot, P., Brink, M., Samos, C. H., Hsieh, J.-C., Wang, Y., Macke, J. P., Andrew, D., Nathans, J. and Nusse, R. (1996). A new member of the frizzled family from Drosophila functions as a Wingless receptor. Nature 382, 225-230.

Brand, A. H. and Perrimon, N. (1993). Targeted gene expression as a means of altering cell fates and generating dominant phenotypes. Development 118, 401-415.

Brunner, E., Peter, O., Schweizer, L. and Basler, K. (1997). pangolin encodes a Lef-1 homolog that acts downstream of Armadillo to transduce the Wingless signal. Nature 385, 829-833.

Cook, D., Fry, M. J., Hughes, K., Sumathipala, R., Woodgett, J. R. and Dale, T. C. (1996). Wingless inactivates glycogen synthase kinase-3 via an intracellular signalling pathway which involves protein kinase C. EMBO J. 15, 4526-4536.

Cox, R. T., Kirkpatrick, C. and Peifer, M. (1996). Armadillo is required for adherens junction assembly, cell polarity, and morphogenesis during Drosophila embryogenesis. J. Cell Biol. 134, 133-148.

DiNardo, S., Kuner, J. M., Theis, J. and O'Farrell, P. H. (1985). Development of the embryonic pattern in D. melanogaster as revealed by the accumulation of the nuclear engrailed protein. Cell 43, 59-69.

DiNardo, S., Sher, E., Heemskerk-Jongens, J., Kassis, J. A. and O'Farrell, P. (1988). Two-tiered regulation of spatially patterned engrailed gene expression during Drosophila embryogenesis. Nature 332, 604-609.

Funayama, N., Fagatto, F., McCrea, P. and Gumbiner, B. M. (1995). Embryonic axis induction by the Armadillo repeat domain of $\beta$-catenin: evidence for intracellular signaling. J. Cell Biol. 128, 959-968.

Hayashi, S., Rubinfeld, B., Souza, B., Polakis, P., Wieschaus, E. and Levine, A. (1997). A Drosophila homolog of the tumor suppressor gene adenomatous polyposis coli down-regulates $\beta$-catenin but its zygotic expression is not essential for the regulation of Armadillo. Proc. Nat. Acad. Sci. USA 94, 242-247.

Hochstrasser, M. (1996). Protein degradation or regulation: Ub the judge. Cell 84, 813-815.

Huber, O., Korn, R., McLaughlin, J., Ohsugi, M., Herrmann, B. G. and Kemler, R. (1996). Nuclear localization of $\beta$-catenin by interaction with transcription factor LEF-1. Mech. Dev. 59, 3-10.

Kawanishi, J., Kato, J., Sasaki, K., Fujii, S., Watanabe, N. and Niitsu, Y. (1995). Loss of E-cadherin-dependent cell-cell adhesion due to mutation of the $\beta$-catenin gene in a human cancer cell line, HSC-39. Mol. Cell. Biol. 15 $1175-1181$

Korinek, V., Barker, N., Morin, P. J., van Wichen, D., de Weger, R., Kinzler, K., Vogelstein, B. and Clevers, H. (1997). Constitutive transcriptional activation by a $\beta$-catenin-Tcf complex in APC-/- colon carcinoma. Science 275, 1784-1787.

Molenaar, M., van de Wetering, M., Oosterwegel, M., Peterson-Maduro, J., Godsave, S., Korinek, V., Roose, J., Destrée, O. and Clevers, H. (1996). XTcf-3 transcription factor mediates $\beta$-catenin-induced axis formation in Xenopus embryos. Cell 86, 391-399.

Morin, P. J., Sparks, A. B., Korinek, V., Barker, N., Clevers, H., Vogelstein, B. and Kinzler, K. (1997). Activation of $\beta$-catenin/Tcf signaling in colon cancer by mutations in $\beta$-catenin or APC. Science 275, 1787-1790.

Munemitsu, S., Souza, B., Albert, I., Rubinfeld, B. and Polakis, P. (1995). Regulation of intracellular $\beta$-catenin levels by the adenomatous polyposis coli (APC) tumor suppressor protein. Proc. Nat. Acad. Sci. USA 92, 30463050 .

Munemitsu, S., Albert, I., Rubinfeld, B. and Polakis, P. (1996). Deletion of an amino-terminal sequence stabilizes $\beta$-catenin in vivo and promotes hyperphosphorylation of the adenomatous polyposis coli tumor suppressor protein. Mol. Cell. Biol. 16, 4088-4094.

Noordermeer, J., Johnston, P., Rijsewijk, F., Nusse, R. and Lawrence, P. A. (1992). The consequences of ubiquitous expression of the wingless gene in the Drosophila embryo. Development 116, 711-719.

Nüsslein-Volhard, C. and Wieschaus, E. (1980). Mutations affecting segment number and polarity in Drosophila. Nature 287, 795-801.

Orsulic, S. and Peifer, M. (1996a). Wingless lands at last. Current Biology 6 , 1363-1367.

Orsulic, S. and Peifer, M. (1996b). An in vivo structure-function analysis of armadillo, the $\beta$-catenin homologue, reveals both separate and overlapping regions of the protein required for cell adhesion and wingless signaling. $J$. Cell Biol. 134, 1283-1301.

Pai, L.-M., Kirkpatrick, C., Blanton, J., Oda, H., Takeichi, M. and Peifer, M. (1996). Drosophila $\alpha$-catenin and E-cadherin bind to distinct regions of Drosophila Armadillo. J. Biol. Chem. 271, 32411-32420.

Parr, B. A. and McMahon, A. P. (1994). Wht genes and vertebrate development. Curr. Opin. Genet. Dev. 4, 523-528.

Peifer, M. (1993). The product of the Drosophila segment polarity gene armadillo is part of a multi-protein complex resembling the vertebrate adherens junction. J. Cell Sci. 105, 993-1000.

Peifer, M. and Wieschaus, E. (1990). The segment polarity gene armadillo encodes a functionally modular protein that is the Drosophila homolog of human plakoglobin. Cell 63, 1167-1178.

Peifer, M., Rauskolb, C., Williams, M., Riggleman, B. and Wieschaus, E. (1991). The segment polarity gene armadillo affects the wingless signaling pathway in both embryonic and adult pattern formation. Development 111, 1028-1043.

Peifer, M., Sweeton, D., Casey, M. and Wieschaus, E. (1994a). wingless signal and Zeste-white 3 kinase trigger opposing changes in the intracellular distribution of Armadillo. Development 120, 369-380.

Peifer, M., Pai, L.-M. and Casey, M. (1994b). Phosphorylation of the Drosophila adherens junction protein Armadillo: roles for Wingless signal and Zeste white-3 kinase. Dev. Biol. 166, 543-556.

Pierce, S. B. and Kimelman, D. (1995). Regulation of Spemann organizer function by the intracellular kinase Xgsk-3. Development 121, 755-765.

Riese, J., Yu, X., Munnerlyn, A., Eresh, S., Hsu, S.-C., Grosschedl, R. and Bienz, M. (1997). LEF-1, a nuclear factor coordinating signalling inputs from wingless and decapentaplegic. Cell 88, 777-787.

Robbins, P. F., El-Gamil, M., Li, Y. F., Kawakami, Y., Loftus, D., Appella, E. and Rosenberg, S. A. (1996). A mutated beta-catenin gene encodes a melanoma-specific antigen recognized by tumor infiltrating lymphocytes. $J$. Exp. Med. 183, 1185-92.

Rubinfeld, B., Albert, I., Porfiri, E., Fiol, C., Munemitsu, S. and Polakis, P. (1996). Binding of GSK- $\beta$ to the APC/ $\beta$-catenin complex and regulation of complex assembly. Science 272, 1023-1026.

Rubinfeld, B., Robbins, P., El-Gamil, M., Albert, I., Porfiri, E. and Polakis, P. (1997). Stabilization of $\beta$-catenin by genetic defects in melanoma cell lines. Science 275, 1790-1792.

Sampedro, J., Johnston, P. and Lawrence, P. A. (1993). A role for wingless in the segmental gradient of Drosophila? Development 117, 677-687.

Siegfried, E., Wilder, E. and Perrimon, N. (1994). Components of wingless signaling in Drosophila. Nature 367, 76-80.

Stambolic, V., Ruel, L. and Woodgett, J. R. (1996). Lithium inhibits glycogen 
synthase kinase-3 activity and mimics Wingless signaling in intact cells. Curr. Biol. 6, 1664-1668.

van de Wetering, M., Cavallo, R., Dooijes, D., van Beest, M., van Es, J., Loureiro, J., Ypma, A., Hursh, D., Jones, T., Bejsovec, A., Peifer, M., Mortin, M. and Clevers, H. (1997). Armadillo co-activates transcription driven by the product of the Drosophila segment polarity gene $d T C F$. Cell $\mathbf{8 8}$, 789-799.

van den Heuvel, M., Nusse, R., Johnston, P. and Lawrence, P. A. (1989). Distribution of the wingless gene product in Drosophila embryos: a protein involved in cell-cell communication. Cell 59, 739-749.

van Leeuwen, F., Harryman-Samos, C. and Nusse, R. (1994). Biological activity of soluble wingless protein in cultured Drosophila cells. Nature $\mathbf{3 6 8}$, 342-344.

Vleminckx, K., Wong, E., Guger, K., Rubinfeld, B., Polakis, P. and Gumbiner, B. M. (1997). Adenomatous polyposis tumor suppressor protein has signaling activity in Xenopus embryos resulting in the induction of an ectopic dorsoanterior axis. J. Cell Biol. 136, 411-420.

Whitehead, I., Kirk, H. and Kay, R. (1995). Expression cloning of oncogenes by retroviral transfer of cDNA libraries. Mol. Cell. Biol. 15, 704-710.
Wieschaus, E. and Nüsslein-Volhard, C. (1986). Looking at embryos. In Drosophila, A Practical Approach, (ed. D. B. Roberts), 199-228. IRL Press, Oxford, England.

Yanagawa, S., van Leeuwen, F., Wodarz, A., Klingensmith, J. and Nusse, R. (1995). The dishevelled protein is modified by wingless signaling in Drosophila. Genes Dev. 9, 1087-1097.

Yoffe, K. B., Manoukian, A. S., Wilder, E. L., Brand, A. H. and Perrimon, N. (1995). Evidence for engrailed-independent wingless autoregulation in Drosophila. Dev. Biol. 170, 636-650.

Yost, C., Torres, M., Miller, J. R., Huang, E., Kimelman, D. and Moon, R. T. (1996). The axis-inducing activity, stability, and subcellular distribution of $\beta$ catenin is regulated in Xenopus embryos by glycogen synthase kinase. Genes Dev. 10, 1443-1454.

Zecca, M., Basler, K. and Struhl, G. (1996). Direct and long-range action of a wingless morphogen gradient. Cell 87, 833-844.

(Accepted 25 March 1997) 